\title{
SPATIO-TEMPORAL MODEL FOR PREDICTING COVID19 CASES IN INDONESIA
}

IMA $(1,1)$ and a Bayesian Poisson Regression model.

\author{
Nanda Rizqia Pradana Ratnasari*, Vita Rosiana Dewi \\ *Indonesia International Institute for Life Science
}

*nandarizqiapradana@gmail.com

\begin{abstract}
Objective: Spatio-temporal modelling is a method used for data which has spatial (area) and temporal (time) property. Confirmed cases of Covid19 in each province Indonesia were recorded from March $2^{\text {nd }}$ to September $15^{\text {th }}, 2020$. The spatio-temporal model in this study are split into two parts which are $\operatorname{ARIMA}(p, d, q)$ for the temporal pattern and Bayesian Poisson regression to explain the spatial pattern.

Method: Data for the study was obtained from Data Repository of Indonesian National Board for Disaster Management - Indonesia Task Force for Covid-19 Rapid Response (Gugus tugas Percepatan penangana Covid19) official website which are an opened source data. The Rstudio, Arcgis and excel was used to carry out the statistical analysis involved in the investigation. In the temporal analysis, data was assumed to have an increasing trend and to create a stationary series, an integrated method was conducted. Box-Jenskin and Ljung-Box method was taken in parameter estimation and model identification process. For the spatial analysis, a Bayesian Poisson Regression is fitted to the dataset with Metropolis algorithm.

Result: Model $\operatorname{IMA}(1,1)$, in general, can explain he increasing trend in the Covid19 confirmed cases in Indonesia. This model can define that the case number at the particular time is affected by the moving average at lag-1. Meaanwhile, a Bayesian Poisson Regression can elaborate spatial pattern in the data. The fitted model shows that the confirmed cases at particular province is also affected by the population density at those provinces. As there are some limitation in the data and method applied in the study, further analysis and research are needed.
\end{abstract}

\section{INTRODUCTION}

At the late December 2019, the first case of a new virus causing resent outbreak called Covid19 was found in Wuhan, China. Up until now, the number of positive cases is still growing and the virus expand to number of countries over the world. The number of confirmed cases and death of this disease significantly rise over time with a high number of community transmission. It also becomes a complicated problem for numbers of countries as there is no global standard measurements to manage and to account the pandemic $[1,2,5]$. As it is hard to control the spread of the virus, many countries have dealt with new challenges related to public health, medications and social-economics, and it takes decades to recover all these matters [3]. World Health Organization (WHO) classifies this pandemic as a public health of international concern [5]. Government in most countries implement new rules and policy to prevent the increasing number of cases and also the spreading news 
related to the virus that can affect and worsen the situation in society. Some studies and investigation have been conducted to recover these worrying situation [4].

Considering how the virus spread, exploring the spatio-temporal characteristic and pattern of Covid19 data can be an effective way to prevent and control the pandemic [5]. Understanding the spatial spread of the disease becomes a critical method to predict local and global outbreaks as well as to develop public policy which will be effective for each area [6]. The analysis describes the epidemical characteristics of the disease, including distribution and association. The Spatio-temporal model and analysis can identify the temporal trends and changes of daily new confirmed cases that elaborate with the spatial pattern of the positive cases [5]. Also, the Spatio-temporal analysis can explore the existence of relationship between factors in the data based on area in which the factors were recorded [7]. Meanwhile, the temporal part can explain the actual condition of the disease counted over the period of the outbreak [8].

In Indonesia, high number of recorded positive cases is allegedly caused by the large number of population and high mobility rate. Moreover, low numbers of testing also become a supporting factor of increasing trend of the confirmed cases. Indonesia may also face a threat regarding vast quantity of underreported cases which can lead to improper policy [8, 9]. Furthermore, most cases show that some people who carry the disease do not have particular symptoms. This may escalate the confirmed positive cases as people think that restriction in social interaction is no not necessary [10]. In order to account the problems, Indonesian Government has applied new regulations such as forming The Indonesia Task Force for Covid-19 Rapid Response, limiting people's mobility in public areas [11].

Some studies have been conducted to find the pattern of the cases. However, it cannot really explain the actual data over the time [10]. Furthermore, the variability in each area, sometimes, cannot be elaborated easily. The area heterogeneity becomes a factor that needs to be explored thoroughly [12]. Therefore, to elaborate all the possibility that may happen in Indonesia caused by the disease, this paper will provide an explanation, as well as models that can illustrate the temporal and spatial aspect of the outbreak.

\section{METHODOLOGY}

\section{DATA AND DATA SOURCE}

Dataset used in this study was taken from Indonesian National Board for Disaster Management - Indonesia Task Force for Covid-19 Rapid Response official website on September $15^{\text {th }}, 2020$. The data recorded some information (variables) related to Covid19 in Indonesia such as number of confirmed cases, death, recovered patients, examined specimens, etc. The data are updated daily since from the first discovered cased on March $2^{\text {nd }}, 2020$. Furthermore, variables used in this analysis is the daily number of confirmed cases of Covid19 in Indonesia.

To answer the purposes of this research, there are two difference type of dataset downloaded from the official website. The first one is the temporal data which consist of daily cases reported in Indonesia. And the second data is a spatial data that inform the cumulative cases per September $15^{\text {th }}$ for each province in Indonesia.

\section{TEMPORAL ANALYSIS}

Theoretically, ARIMA model is one of the best models for predicting time series data since this model consist of three characteristics. They are Autoregressive, Integrated and Moving 
Average. This model is suitable for explaining univariate time series data. To perform ARIMA model, some steps are applied, starting from fitting an appropriate model, estimating model parameters for the chosen orders and verifying the model $[8,13]$.

ARIMA model is applied at broad areas such as epidemiology and public health. In those areas, time series model has been applied extensively since it is very beneficial as it could predict actual and future cases. Number of forecasting in epidemiology for several cases such as influenza and dengue has been conducted using ARIMA model [8].

ARIMA model assumes that values of variables in particular time is affected by the values of past observations. While the $\operatorname{AR}(p)$ expresses a linear combination of past events with order $p$, the $\operatorname{MA}(q)$ uses past errors as the explanatory variables [13]. Integrated characteristics is utilized to create a stationary process obtained by differencing a nonstationary process. The process is developed according to the original Box-Jenkins methodology [14].

The formulation for $\operatorname{ARIMA}(p, d, q)$ is expressed by the following equations:

$$
\begin{aligned}
& y_{t}=\emptyset_{1} y_{t-1}+\emptyset_{2} y_{t-2}+\cdots+\emptyset_{p} y_{t-p}+\omega_{t}=\sum_{i=1}^{p} \emptyset_{i} y_{t-i} \\
& y_{t}=\theta_{1} \omega_{t-1}+\theta_{2} \omega_{t-2}+\cdots+\theta_{p} \omega_{t-p}+\omega_{t}=\sum_{j=1}^{q} \theta_{j} \omega_{j-q}
\end{aligned}
$$

where $y_{t}$ is stationary process; $\emptyset_{1}, \emptyset_{2}, \ldots, \emptyset_{p}$ are constant for AR model with $p$ order, and $\omega_{t}$ is white noise series with zero mean and variance $\sigma_{\omega}^{2}$. The $\operatorname{AR}(p)$ model predict $y_{t}$ based on the previous events $y_{1}, y_{2}, \ldots, y_{t-1}$. Meanwhile, the $\operatorname{MA}(q)$ explains $y_{t}$ by a linear combination of the $q$ white noise $\omega_{1}, \omega_{2}, \ldots, \omega_{t-q}$.

The combination of autoregressive order $p$ and moving average order $q$ forms a new model called $\operatorname{ARMA}(p, q)$ with formula:

$$
y_{t}=\delta+\sum_{i=1}^{p} \emptyset_{i} y_{t-i}+\sum_{j=1}^{q} \theta_{j} \omega_{j-q}+\omega_{t}
$$

The stationary $\operatorname{ARMA}(p, q)$ process after being differenced $d$ times is notated by $\operatorname{ARIMA}(p, d$, q):

$\Delta^{d} y_{t}=\delta+\emptyset_{1} \Delta^{d} y_{t-1}+\cdots+\emptyset_{p} \Delta^{d} y_{t-p}+\theta_{1} \omega_{t-1}+\cdots+\theta_{p} \omega_{t-p}+\omega_{t}$

where $\Delta^{d}$ denoted $d$-th difference time series.

In time series processes, plots of autocorrelation function (ACF) and partial autocorrelation function (PACF) are used to identify the order parameter of AR and MA model. The $\operatorname{AR}(p)$ parameters are obtained when ACF exhibits a tendency lying down quickly whereas the PACF show a spike. In the contrary, the $\mathrm{MA}(q)$ parameters are obtained when ACF tends to show spike and the PACF exhibits a lie down lines [14].

The time series ARIMA model should be stationary and stochastic sequence should have zero mean to produce a valid forecasting model. If there is sign of nonstationary, the data should be differenced using an integrated model [8].

\section{(1) Stationarity}


Stationary in time series is interpreted as a condition where the data does not have a trend or seasonality. The white noise of the time series data will be stationary if it does form any specific pattern or looks constant over the time[8,14]. The stationarity of a data could be identified with Box-Jenkins. Box-Jenkins method determines stationarity of the data when all the root of the characteristic equations fall outside the circle [13]. Also, Dickey-Fuller test and Kwiat-kowski-Phillips-Schmidt-Shin (KPSS) test are methods used to check the stationary of time series data. Those tests can be conducted using $\mathrm{R}$ software $[8,13]$.

\section{(2) Order Identification}

Identification can be conducted by evaluating the order of autocorrelation function (ACF) and partial autocorrelation function (PACF). Subsequently, order of the model in general is determined from 0 to 2 since the model is expected to be as simple as possible to fulfil the parsimony theorem. The quality of statistical model and the prediction of the error is given by AIC (Akaike Information Criterion) [8]. The optimal model order is chosen by evaluating the AIC measurement. Furthermore, to check whether the white noise obtained from the residuals is white noise, Ljung-Box test can be used [13].

\section{(3) Estimation and Diagnosis}

Among all candidate models, the best and appropriate model is diagnosed using residual errors. The errors represent the difference between actual data and the prediction of the fitted model. The errors are expected to be white noise which is identified using Ljung-Box method. White noise error means that the residuals are random and do not have a significant correlation [15].

\section{(4) Forecasting}

The final process of performing time series model is to forecast the actual data and the future events. The appropriate model may have forecasted data that close enough to the actual observation $[8,13]$.

\section{SPATIAL ANALYSIS}

Spatial data are data that have a spatial component. This means that the data are correlated geographically between region where the data are recorded. Spatial data types provide a fundamental abstraction for modelling the geometric structure of objects in space as well as their relationships, properties, and operations [16]. Spatial association of a collection of data can be detected using Moran's $I$ statistics that elaborate data with various types of neighbourhoods [17]. Moran's I statistics measures the spatial autocorrelation in the data which can be calculated using the equation below:

$$
I=\frac{n \sum_{i j} W_{i j}\left(Y_{i}-\bar{Y}\right)\left(Y_{j}-\bar{Y}\right)}{\Sigma_{i \neq j} W_{i j} \Sigma_{i}\left(Y_{i}-\bar{Y}\right)^{2}}
$$

where $i$ and $j$ are the region indexes and $W_{i j}$ indicates the adjacency between area $i$ and area $j$. And, $Y_{i}$ and $Y_{j}$ denotes new confirmed cases and $\bar{Y}$ is the average number of new cases [6]. The value of Moran's I statistics explains spatial autocorrelations in the data. The value of these term will range from negative to positive values. The zero value represents no autocorrelation in the data, the negative value indicates the clustering of similar values, whereas a negative Moran's $I$ value indicated the clustering of dissimilar values $[6,17]$.

Moran's $I$ index is a measure of spatial autocorrelation or independency of the data that can explore the spatial structure of disease [5]. Autocorrelation in the spatial data indicated 
by Moran's $I$ value will show significant variation caused by spatial heterogeneity of data [18].

\section{Bayesian Poisson Regression}

The interest of this study was to predict the number of covid19 positive cases for each province in Indonesia. As the number of confirmed cases is counted data, the Poisson Regression might be the appropriate model applied on the available dataset. As the statement given in the introduction said that the confirmed cases might be caused by the number of population, then a population density for each province in Indonesia became the dependent variable. However, an Ordinary Least Square(OLS) approach fitted on the available dataset might be not able to account the spatial variability on the dataset. Therefore, Bayesian Poisson Regression could be the better option to be fitted on the dataset [21].

The Bayesian Poisson Regression derived from the OLS Poisson Regression. The Poisson regression model with a log-link given in the following equation

$$
\log (E(\boldsymbol{Y} \mid \boldsymbol{x}))=\log \left(\theta_{x}\right)=\beta_{0}+\beta_{1} x
$$

this means that $\boldsymbol{Y} \mid \boldsymbol{x} \sim$ Poisson $\left(\exp \left[\boldsymbol{\beta}^{\boldsymbol{T}} x\right)\right.$

In a Bayesian setting, we need to assign prior distributions to the parameters $\beta$ to obtain posterior distribution. The posterior distribution of $\beta$, given the training data $\{\mathbf{X}, \mathbf{y}\}$, is computed with Bayes' rule

$$
\frac{p(\boldsymbol{\beta} \mid \boldsymbol{X}, \boldsymbol{Y}) p(\boldsymbol{\beta}) p\left(b^{*}\right)}{\int p(\boldsymbol{Y} \mid \boldsymbol{X}, \boldsymbol{\beta}) p(\boldsymbol{\beta}) d(\boldsymbol{\beta})}
$$

However, even we could determine the prior distribution of the Bayesian Poisson Regression, it is is often hard to calculate due to the integral in the denominator. As the posterior distribution of the Bayesian Poisson Regression is analytically unobtainable, a Monte-Carlo approximations are applied to obtain the posterior distribution. The approach used to obtain the closed form of the posterior analysis is Metropolis algorithm [19]. The Metropolis-Hastings sampling algorithm is a class of Markov Chain Monte Carlo (MCMC) method whose main idea is to generate a Markov Chain $\left\{X_{t} \mid \mathrm{t}=0,1,2, \ldots\right\}$ such that its stationary distribution is the target distribution. In this analysis, the stationary distribution is the posterior distribution of the parameters $\beta$. [21]

\section{Statistical Analysis}

The analysis conducted in the study use Rstudio software with timeseries package for analysing time series data and using Arcgis software for elaborating spatial data. For the temporal analysis, first step established was defining the stationary of the data, which used Dickey-Fuller test. The series and correlation of the data were plotted to see the data sequences and to identify orders of the model. Estimation of the parameters was using maximum likelihood method that was provided in the software. Model fitting tests were carried out to get estimated parameters and significant level, including the standard error, log-likelihood value, AIC and residual errors. Meanwhile for the spatial data, the analysis can be conducted by discovering Spatial Patterns using Local Moran's I Index. The first step is calculating the index value, then determining the expected and variance value of Moran's I 
value to obtain z-value. The final calculation of z-value can be used to find decision of null hypothesis stating independency of spatial locations.

\section{RESULTS AND DISCUSSION}

\section{TEMPORAL ANALYSIS}

There were two different types of dataset used in this study. Those are "daily confirmed cases" for time series analysis purposes and "confirmed cases recorded for each province" data for spatial analysis purposes. The data was recorded from March $2^{\text {nd }}$ when the first case was found in Depok to September $15^{\text {th }}, 2020$. The time series data was analysed using $\operatorname{ARIMA}(p, d, q)$ model, whereas the spatial data was analysed using Moran's I to check the autocorrelation of the spatial component.

The time series data consist of 198 rows of daily confirmed cases and the spatial data consist of 34 rows cumulative confirmed cases recorded for each province.

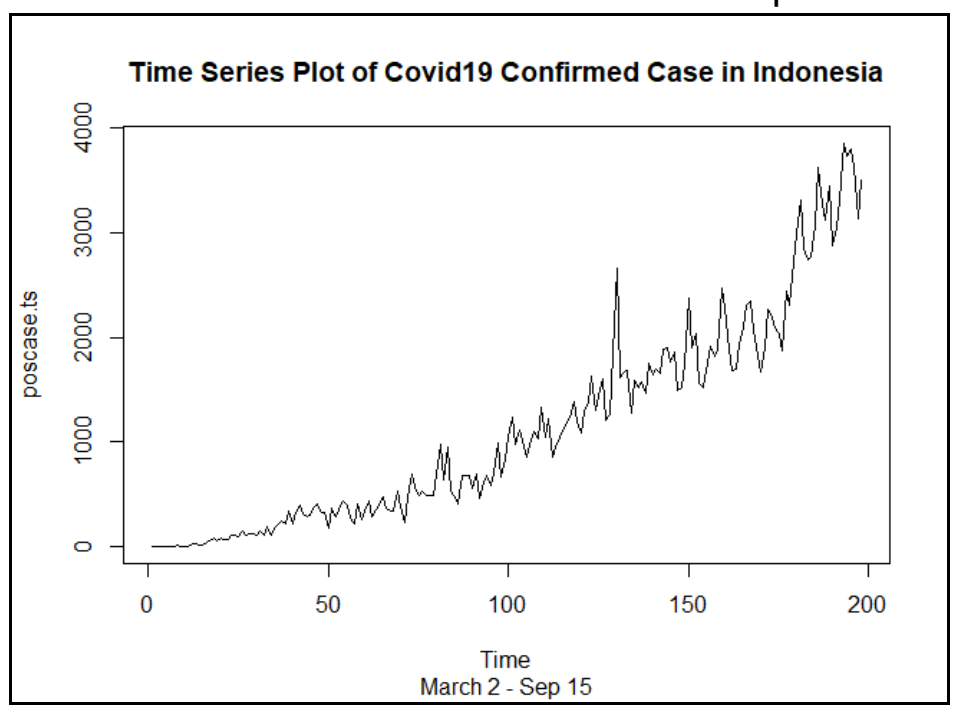

Figure 1. Plot of Covid19 Confirmed Case Data

Figure 1 shows that the time series data of Covid19 confirmed cases in Indonesia has an increasing trend with no sign of cyclical or seasonal pattern. The minimum values were zero where there was not any discovered case on few days of first week of observations. The confirmed cases hit the highest number on September 10, 2020 of 3861 persons. This could be caused by the eased restriction policy, leading to the community freely doing their activities. During the pandemic, the average value of confirmed cases was around 1137 which could be said as a very high number.

However, after $100^{\text {th }}$ day, the confirmed cases hit another peak and the number has been high and have tendency to increase over time. Loosening the restriction policy while at the same time increasing the test rate of the virus on people are the reasons why the number was rising.

The first step in the time series data is checking the stationary of the data. Dickey-Fuller test was conducted for this purpose. 


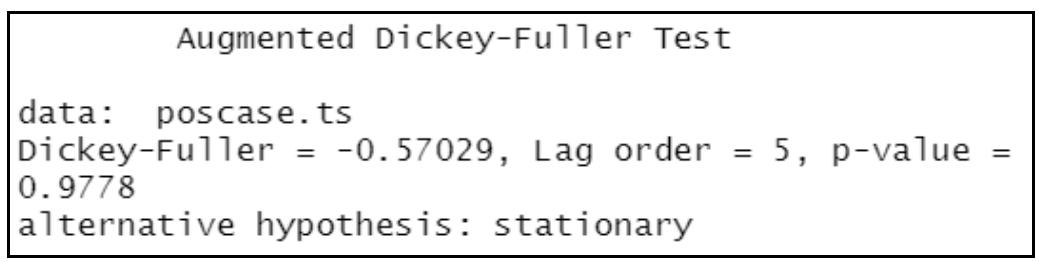

Figure 2. Output of Dickey-Fuller Test for Actual Data

Figure 2 represents output from Dickey-Fuller test conducted in Rstudio. The null hypothesis states that the data is nonstationary. As the $p$-value is higher than 0.05 , then it indicated that the confirmed cases data is not stationary. Therefore, for further analysis, the data should be transformed using difference.

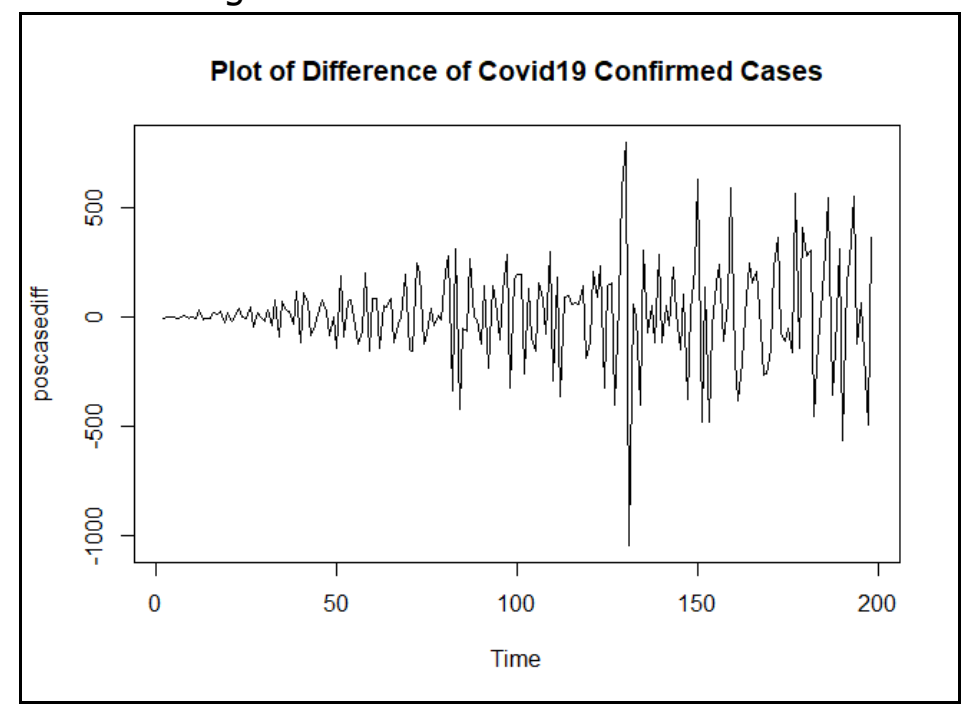

Figure 3. Plot of Difference of Covid19 Confirmed Case Data

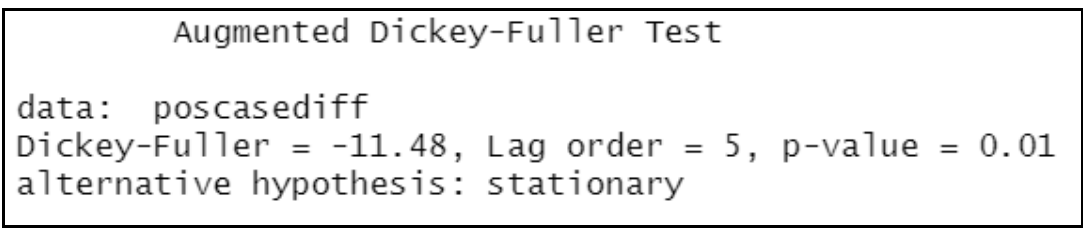

Figure 4. Output of Dickey-Fuller Test for Differenced Data

The transformed data was plotted and it is displayed in Figure 3. The plot indicated that the data no longer has a trend. This was further confirmed by the Dickey-Fuller test shown in Figure 4 that suggested the data is stationary with p-value equals to 0.01 . Since the pvalue less than 0.05 , this means that the data is stationary and could be analysed using integrated $d=1$. 


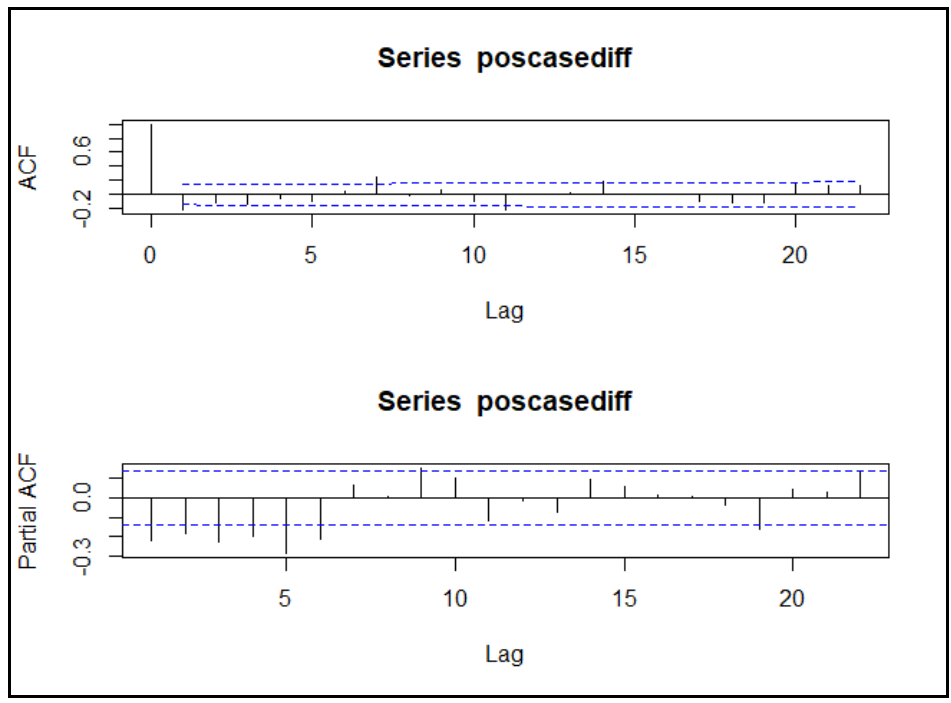

Figure 5. ACF and PACF Plot of Covid19 Confirmed Cases

The order of ARIMA model could be identified using plot ACF and PACF. Figure 5 shows the PACF plots lying down with sinus wave and the ACF plot having tendency to show a spike at the first lag. Based on those plots, the model could be determined using MA model with order $p=1$ and integrated order $d=1$. Therefore, the fitted model could be recognized as $\operatorname{IMA}(1,1)$.

Based on the model identification, the parameters are estimated using Maximum Likelihood (ML) method. The parameter of integrated IMA $(1,1)$ for Covid19 confirmed cases was -0.4370 with standard error equal to 0.1108 . The AIC obtained from the model estimation was 2682.98 .

Figure 6 shows that the residual plots computed by subtracting the actual data and the fitted model forecasting values. The residuals indicated a stationary pattern since it constant around and it does not have significant correlation with other lags. The residuals correlate only with lag $=0$ which means that it relates to itself.

Figure 7 shows the Ljung-Box plot to check whether the residuals from the fitted model are white noise. The figure exhibits that $p$-values for Ljung-Box statistics at lag zero is not significant and above the line. This indicates that the model fitting is appropriate at first lag.

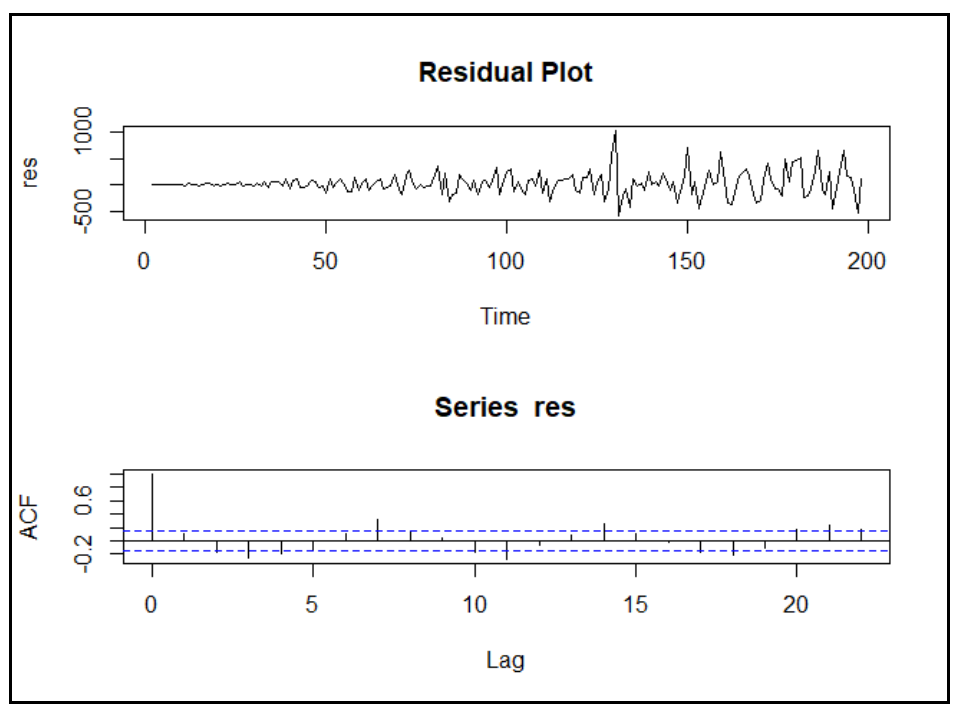

Figure 6. Residuals Plot 


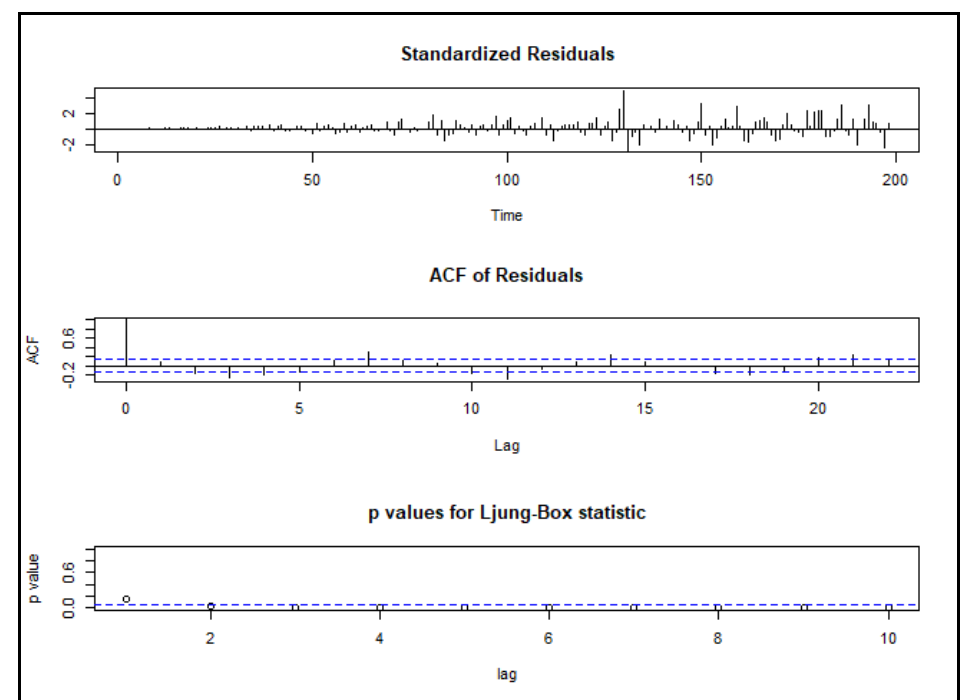

Figure 7. Residuals Plot

Forecasting is needed to check whether the model can represent the actual values. Based on the model identified using $\operatorname{IMA}(1,1)$, the prediction of the estimated values can be forecasted. Figure 7 shows that the predicted model could estimate actual data both the pattern and points.

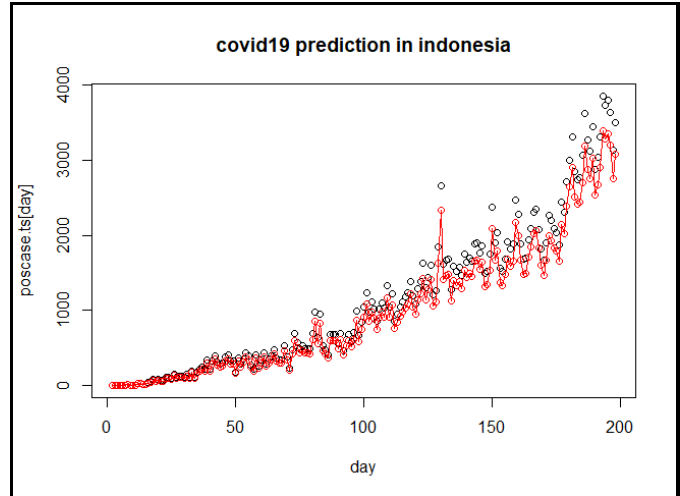

(a)

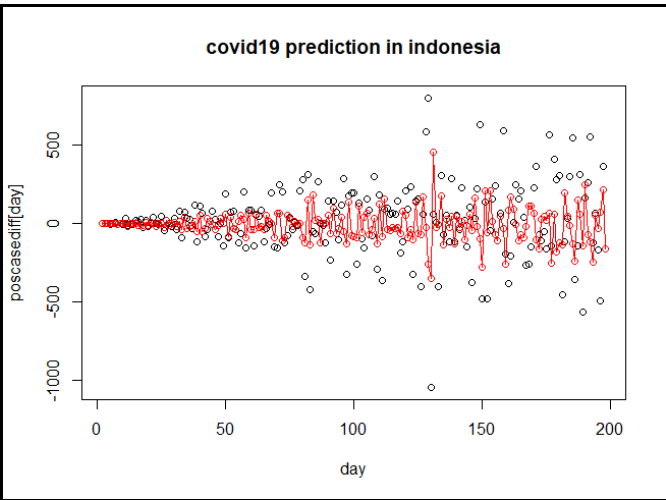

(b)

Figure 8. Prediction of Covid19 Confirmed Cases based on model $\operatorname{IMA}(1,1)$ for (a) actual data and (b) integrated data

\section{SPATIAL ANALYSIS}

The Figure 9 shows temporal patterns of cumulative new confirmed cases in each province in Indonesia. The graph exhibits that areas around Jakarta have high number of confirmed cases compared to the other provinces in Indonesia followed by East Java and other areas in Java island. Based on the data obtained from Indonesia Bureau of Statistics [20], areas with high cases related with high population density. 


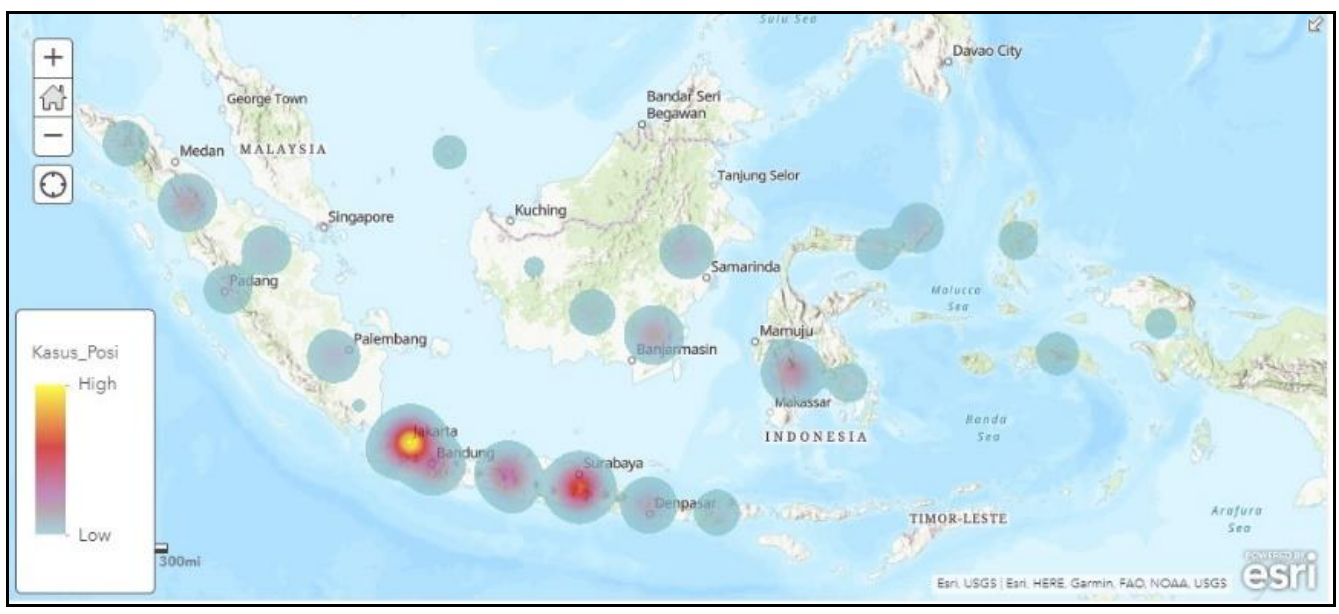

Figure 9. Spatial Plot of Confirmed (Positive) Cases

Moran's I index is a measure of spatial analysis dependency that is used to explore the spatial structure of the incidence rate of the epidemic in the study area. The result from Moran's I analysis explain that there are four type of spatial pattern. The pattern are highhigh $(\mathrm{HH})$ and low-low (LL) cluster indicating several adjacent areas with relatively high(low) value incidence of the epidemic, as well as, high-low (HL) and low-high (LH) outliers which indicate high (low) value surrounded by low (high) value. The Covid19 data in Indonesia presents that most of area in Java has $\mathrm{HH}$ cluster, especially area around Jakarta and East Java. The area with LL cluster is formed in province with relatively low number of disease such as Papua and Maluku. Meanwhile, outliers occur in some province in Sumatra and Kalimantan.

Since the preliminary spatial analysis suggest that there is a correlation between the number of confirmed cases and population density, a regression model involving those two variables are fitted. The number of confirmed cases is counted data, hence, a Poisson regression with population density as its covariate are fitted to the dataset. After fitting the model, the obtained summary results given follow;

Call:

glm(formula $=$ Kasus_Posi $\sim$ Kepadatan_Pend, family = "poisson",

data $=$ data 1$)$

Deviance Residuals:

Min 1Q Median 3Q Max

$\begin{array}{lllll}-90.115 & -69.682 & -31.285 & 1.977 & 291.891\end{array}$

Coefficients:

Estimate Std. Error $z$ value $\operatorname{Pr}(>|z|)$ 
(Intercept) $\quad 8.477 \mathrm{e}+00 \quad 2.503 \mathrm{e}-03 \quad 3387.1<2 \mathrm{e}-16 * * *$

Kepadatan_Pend $1.636 \mathrm{e}-04 \quad 3.185 \mathrm{e}-07 \quad 513.7<2 \mathrm{e}-16$ ***

Figure 10. Parameters for Spatial Analysis

The summary result given above suggest that population density significantly affect the number of confirmed cases because the related $p$-value smaller than 0.05 . After fitting the model, the residuals was further analysed to see whether they are correlated with each other.

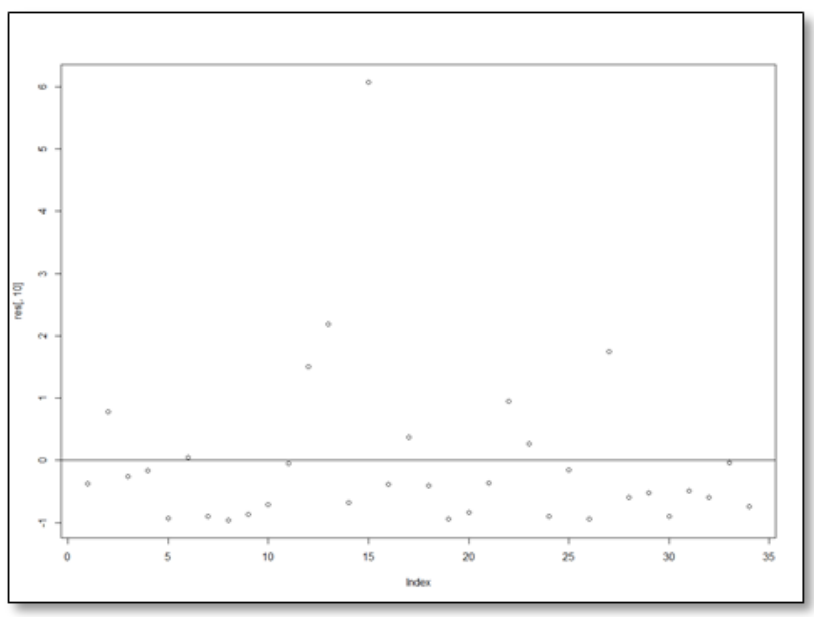

Figure 11. Poisson Regression Residuals

Figure 10 suggest that almost all the residuals mostly crowd under zero which indicate that the model is underdispersion.

The underdispered model might be caused by the spatial correlation. Hence, to getting rid the spatial correlation on the dataset, a Bayesian Poisson Regression is fitted to the dataset with Metropolis algorithm. After performing the Bayesian model, a diagnostics check is performed to make sure that the Bayesian estimated model can be used for predicting the number of new cases in Indonesia. If the draws from the Metropolis algorithm simulation are convergence and independent, then the draws produce reliable posterior estimates for the unknown parameters in the fitted model. In order to see the stationary of the samples, a trace coefficient $\beta_{j}$ the ACF plot are constructed. It is shown in Figure 11.

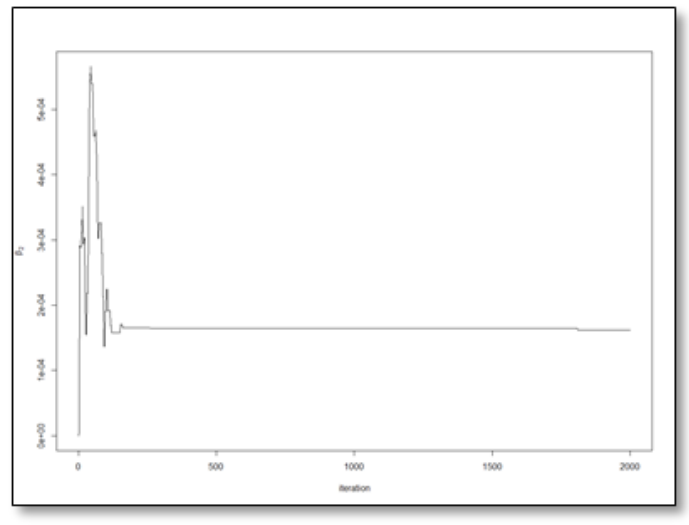

Figure.12 Trace plot for the posterior $\beta$ coefficient

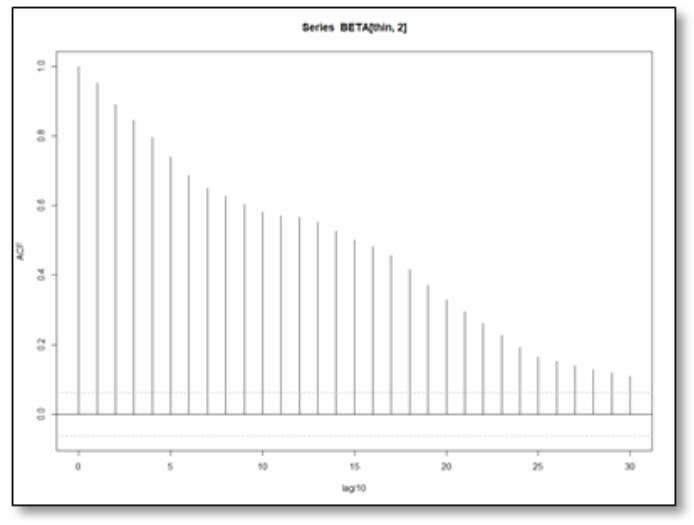

Figure 13. The ACF plot for the posterior $\beta$ coefficients

Figure 10 and Figure 11, it can be said that the samples generated by the MCMC simulation using Metropolis algorithm with 2000 draws have not convergence. For the 
second simulation, 10000 draws were generated. However, the diagnostics check for the second simulation are not much different compared to the first simulation. To check the problem, the Bayesian estimated parameters are computed as the following.

$>$ mean $(\mathrm{BETA}[, 1])$

[1] 8.230607

$>$ mean $(B E T A[, 2])$

[1] 0.0001727488

Figure 14. Expected value for spatial model parameters

Based on the results given above, the Bayesian estimators of the fitted model are almost identical to the estimated parameters produced by Ordinary Least Squared method. Hence, the prediction of both fitted model are almost the same and the Bayesian Poisson Regression model does not able to take account the spatial variability.

Limitation on the model happen in process of model specification. The Bayesian Poisson Regression is not the appropriate model to explain the spatial variability of the covid19 dataset in Indonesia. The problem might arise because there is not any spatial autocorrelated effect put on the fitted data. In order to put such effect, the covid19 cases should be measured in areal form. However, the available dataset was recorded in a spatial point form. Hence, to account for the spatial variability arise in covid19 dataset, a spatial analysis with point statistics approach such as Geographically Weighted can be applied into the available dataset.

\section{CONCLUSION}

This research apply spatio-temporal analysis using Covid19 positive confirmed cases in Indonesia recorded from March $2^{\text {nd }}$ when the first case was found in Depok to September $15^{\text {th }}, 2020$ for each province. Regarding the temporal pattern, integrated moving average model, IMA $(1,1)$, can explain the trend of the data. Based on the model identification, the parameters are estimated using Maximum Likelihood (ML) method. The parameter of integrated IMA $(1,1)$ for Covid19 confirmed cases was -0.4370 with standard error equal to 0.1108. The AIC obtained from the model estimation was 2682.98. As there are problem regarding normality, further analysis for time series or temporal analysis need to be conducted. For spatial pattern, a Bayesian Poisson Regression is fitted to the dataset with Metropolis algorithm can explain the data. The Bayesian estimators of the fitted model are almost identical to the estimated parameters produced by Ordinary Least Squared method. Hence, the prediction of both fitted model are almost the same and the Bayesian Poisson Regression model does not able to take account the spatial variability. However, there is a limitation regarding this model since there is not any spatial autocorrelated effect put on the fitted data. In order to put such effect, the covid19 cases should be measured in areal form.

\section{REFERENCE}


[1] Nadeem, S. 2020. Coronavirus COVID-19: Available Free Literature Provided by Various Companies, Journals and Organizations around the World. J Ong Chem Res, 5(1): page 7-13, Document ID: 2020JOCR37, doi:10.5281/zenodo.3722904.

[2] Gennaro, FD., Pizzol, D., Marotta, C. Antunes, M., Racalbuto, V., Veronese, N., and Smith, L. 2020. Coronavirus Diseases (COVID-19) Current Status and Future Perspective: A Narative Review. Int. J. Environ. Res. Public Health, 17, 2690; doi:10.3390/ijerph17082690.

[3] Djalante, R., et al. 2020. Review and analysis of current responses to Covid-19 in Indonesia: Period of January to March 2020. Progress in Disaster Science, 6.

[4] Nuraini, N., Khairudin, K., and Apri, M. 2020. Modeling Simulation of COVID-19 in Indonesia based on Early Endemic Data. Commun. Biomath. SCI., 3(1): page 1-8. doi:10.5614/cbms.2020.3.1.1

[5] Yang, W., Deng, M., Li, C., and Huang, J. 2020. Spatio Temporal Pattern of the 2019-nCov Epidemic at the Country Level in Hubei Province, China. Int. J. Environ. Res. Public Health 2020, 17, 2563; doi:10.3390/ijerph17072563

[6] Kang, D., Choi, H., Kim, J.H., and Choi, J. 2020. Spatial Epidemic Dynamic of The Covid-19 Outbreak in China. International Journal of Infectious Diseases 94 (2020) 96-102.

[7] Briz-Redon, A., and Serrano-Aroca, A. 2020. A spatio-temporal analysis for exploring the effect of temperature on COVID-19 early evolution in Spain. Science of the Total Environment 728 (2020) 138811

[8] Fadly, F., and Sari, E. 2020. An Approach to Measure the Death Impact of Covid19 in Jakarta using Autoregressive Integrated Moving Average (ARIMA). Unnes Journal of Public Health 9(2), page: 108-116.

[9] Jefriando, M. \& Munthe, B.C. 2020. Indonesia virus death toll rises to highest in Asia outside China. Reuters [Online]. Available at: https://www.reuters.com/article/ushealth-coronavirus-indonesia-jakarta/indonesia-virus-death-toll-rises-to-highest-in-asiaoutside-china-idUSKBN21KOLL [Accessed August 24, 2020].

[10]Berawi, M. A. et al. 2020. Tackling the COVID-19 Pandemic: Managing the Cause, Spread, and Impact. International Journal of Technology, 11(2): page. 209-214. doi: 10.14716/ijtech.v11i2.4035.

[11]Djalante, R., et al. 2020. Review and analysis of current responses to Covid-19 in Indonesia: Period of January to March 2020. Progress in Disaster Science, 6.

[12]Giuliani, D., at al. 2020. Modelling and predicting the spatio-temporal spread of COVID-19 in Italy. Research Square. doi: 10.21203/rs.3.rs-20501/v2 https://www.researchgate.net/publication/342616969 Modelling and predicting the spat io-temporal spread of COVID-19 in Italy

[13]Adhikara, R., and Agrawal, R. K. An Introductory Study on Time Series Modelling and Forecasting. https://arxiv.org/ftp/arxiv/papers/1302/1302.6613.pdf

[14]Waeto, S., Chuarkham, K., and Intarasit, A. 2017. Forecasting Time Series Movement Direction with Hybrid Methodolgy. Journal of Probability and Statistics. Volume 2017, ID 3174305. doi: 10.1155/2017/3174305.

[15]Hyndman, R. J. \& Athanasopoulos, G. 2018. Forecasting: Principles and Practice. Melbourne: Monash University Australia.

[16]Schneider, M. 2009. Spatial data Type. Research Gates. doi: 10.1007/978-0-387-399409_354. 
[17]Li, H., Calder, C.A., and Cressie, N. 2007. Beyond Moran's I: testing for spatial dependence based on the spatial autoregressive model. GeogrAnal: 39(4), page: 357-75.

[18] Anselin, L. Local indicators of spatial association-LISA. Geogr. Anal. 1995, 27, 93115.

[19]Hoff, Peter D. 2009. A first Course in Bayesian Statistical Methods. Springer-Verlag. New York

[20] https://www.bps.go.id/indicator/12/141/1/kepadatan-penduduk-menurut-provinsi.html, accessed 10 September 2020

[21] https://rpubs.com/SaraGarcesCespedes/586440, accessed 8 September 2020 